\title{
A note on utility based pricing and asymptotic risk diversification
}

\author{
Bruno BOUCHARD \\ CEREMADE, CNRS, UMR 7534, \\ Université Paris-Dauphine \\ and CREST \\ bouchard@ceremade.dauphine.fr
}

\author{
Romuald ELIE \\ CEREMADE, CNRS, UMR 7534, \\ Université Paris-Dauphine \\ and CREST \\ elie@ceremade.dauphine.fr
}

\author{
Ludovic MOREAU \\ CEREMADE, CNRS, UMR 7534, \\ Université Paris-Dauphine \\ moreau@ceremade.dauphine.fr
}

August 26, 2011

\begin{abstract}
In principle, liabilities combining both insurancial risks (e.g. mortality/longevity, crop yield,...) and pure financial risks cannot be priced neither by applying the usual actuarial principles of diversification, nor by arbitrage-free replication arguments. Still, it has been often proposed in the literature to combine these two approaches by suggesting to hedge a pure financial payoff computed by taking the mean under the historical/objective probability measure on the part of the risk that can be diversified. Not surprisingly, simple examples show that this approach is typically inconsistent for risk adverse agents. We show that it can nevertheless be recovered asymptotically when the number of sold claims goes to infinity and the absolute risk aversion of the agent goes to zero simultaneously. This follows from a general convergence result on utility indifference prices which is valid for both complete and incomplete financial markets. In particular, if the underlying financial market is complete, the limit price corresponds to the hedging cost of the mean payoff. If the financial market is incomplete but the agent behaves asymptotically as an exponential utility maximizer with vanishing risk aversion, we show that the utility indifference price converges to the expectation of the discounted payoff under the minimal entropy martingale measure.
\end{abstract}

Keywords: Utility indifference pricing, diversification, risk aversion, entropy.

Mathematics subject classification (2010): 60H30, 91B16, 91B24, 91B30.

\section{Introduction}

These last years have seen the explosion of the number of liabilities combining pure financial and pure insurancial risks. They typically have the following form: an insurance company sells to the client $i$ a claim with discounted payoff $g^{i}$ paid at maturity $T$ whose value depends on the evolution of some tradable financial assets $S=\left(S_{t}\right)_{t \geq 0}$ and some additional idiosyncratic risk. The $g^{i}$ 's are usually not unconditionally independent, but still independent conditionally to $S$. 
It is (essentially) the case of many variable annuities schemes in which death times or withdrawals policies can be assumed to be independent conditionally to the financial market's behavior, see e.g. [1]. This is also the case for crop revenue insurance schemes that depend on the production yield of the farmer and the market price of the crop, see e.g. [12]. More examples can be found in [11] or [2].

In such a situation, and if the financial market formed by the financials assets $S$ is complete, it is tempting to play on the ability to diversify the conditionally idiosyncratic risks and cover the systemic pure financial risk by dynamically trading on the market. If the $g^{i}$ s are independent and identically distributed given $S$, then the price of each of these contingent claims could be defined as $\bar{p}:=\mathbb{E}^{\mathbb{Q}}[\bar{g}(S)]$ where $\bar{g}(S):=\mathbb{E}\left[g^{i} \mid S\right]$ does not depend on $i$, and $\mathbb{Q}$ denotes the unique martingale measure on the pure financial market (i.e. restricted to $S$ ). The rationality behind this is the following: by an unformal application of the law of large numbers conditionally to $S$, we obtain the convergence $G_{n} / n:=\sum_{i=1}^{n} g^{i} / n \rightarrow \bar{g}(S)$ a.s. for a large number $n$ of sold contracts. In the above, the payoff $\bar{g}(S)$ only depends on $S$ and can thus be hedged dynamically by trading on the (complete) pure financial market. Hence, by replicating the mean payoff $\bar{g}(S)$, we end up with a zero net position in mean (under the initial probability measure $\mathbb{P}$ ).

This solution has been originally proposed by Brennan and Schwartz [5, 6], and then applied several times, in particular in the literature on variables annuities, see e.g. [1], [4], [14] or [15]. However, it seems to ignore the fact that playing with the law of large numbers on the diversifiable part of the risk requires selling a large number of contracts, and therefore may lead to huge positions on the financial market. If the law of large numbers does not operate well enough, then the losses may be leveraged by an unfavorable evolution of the financial market. More generally, the classical central limit theorem that allows to control the asymptotic distribution of the risk in terms of the Gaussian law will in general not apply in this context.

One classical solution for pricing such claims is to use the indifference pricing rule of HodgeNeuberger [13], see e.g. [2] for the exponential utility case. Not surprisingly, it typically does not lead to the price $\bar{p}$ defined as above, see Section 2.2 for trivial counter-examples. However, one should intuitively recover it when the number of sold contracts is large, so that the conditional law of large numbers can operate, and the risk aversion is small.

In this note, we provide sufficient conditions under which the above holds true. Namely, we consider a family of utility functions $\left(U_{n}\right)_{n}$, defined on $\mathbb{R}$, with corresponding absolute risk aversion $\left(r_{n}\right)_{n}$ and indifference prices $\left(n p_{n}\right)_{n}$ for the aggregate claims $\left(G_{n}\right)_{n}$. Under mild assumptions detailed in Section 3.2, we show that $n \rightarrow \infty$ and $n\left\|r_{n}\right\|_{\infty} \rightarrow 0$ implies $p_{n} \rightarrow \bar{p}$, whenever the underlying financial market formed by the liquid financial assets $S$ is complete. This follows from a more general asymptotic result derived in Section 3.1, which provides a formulation for the asymptotic unit price $\lim _{n} p_{n}$ in terms of the sequence of martingales measures minimizing the corresponding dual problems. The latter applies to incomplete financial markets without providing a clear identification of the asymptotic pricing measure, except when $\left(U_{n}\right)_{n}$ behaves asymptotically like a sequence of exponential utility functions. In this case, we show that $p_{n} \rightarrow p^{e}:=\mathbb{E}^{\mathbb{Q}^{e}}[\bar{g}(S)]$ where $\mathbb{Q}^{e}$ is the martingale measure with minimum relative entropy, see Section 3.3. This generalizes to our setting the well-known property that the exponential utility indifference price of a claim converges to the risk neutral price under $\mathbb{Q}^{e}$ for vanishing risk aversion, see [18] and [2]. Note that a similar result is obtained in [2] for the indifference price of the mean payoff $\bar{G}_{n}:=G_{n} / n=\sum_{i=1}^{n} g^{i} / n$ as $n$ goes to infinity and the risk aversion is fixed, which is a completely different situation.

In the following, any assertion involving random variables has to be understood in the a.s. sense. Given a probability measure $\mathbb{Q}$ and a sigma-algebra $\mathcal{G}$, we denote by $L^{1}(\mathbb{Q}, \mathcal{G})\left(\right.$ resp. $\left.L^{\infty}(\mathbb{Q}, \mathcal{G})\right)$ the space of $\mathbb{Q}$-integrable (resp. $\mathbb{Q}$-essentially bounded) random variables that are $\mathcal{G}$-measurable. We omit the argument $\mathbb{Q}$ or $\mathcal{G}$ if it is clearly given by the context. 


\section{Diversification based pricing rules and risk aversion}

In this section, we describe the financial market and elaborate on the relation between diversification and utility indifference pricing.

\subsection{The market model}

From now on, we fix a time horizon $T>0$ to avoid unnecessary technical issues, although for some applications (e.g. mortality/longetivity linked contracts) it should in principle be infinite. We consider a model of a security market which consists of $d$ stocks with price process described by a locally bounded càdlàg semi-martingale $\left(S^{i}\right)_{1 \leq i \leq d}$ defined on some complete filtered probability space $\left(\Omega, \mathcal{F}, \mathbb{F}:=\left(\mathcal{F}_{t}\right)_{0 \leq t \leq T}, \mathbb{P}\right)$, with $\mathbb{F}$ satisfying the usual assumptions and $\mathcal{F}=\mathcal{F}_{T}$. As usual, we normalize the risk free rate to 0 for simplicity, which can always been done by considering discounted values.

A (self financing) strategy is defined as an element $\vartheta=\left(\vartheta^{i}\right)_{1 \leq i \leq d}$ of the set $\Theta$ of $\mathbb{F}$-predictable $S$-integrable processes. Given an initial endowment $x \in \mathbb{R}$ and a strategy $\vartheta \in \Theta$, the induced wealth process $X^{x, \vartheta}=\left(X_{t}^{x, \vartheta}\right)_{0 \leq t \leq T}$ is given by

$$
X_{t}^{x, \vartheta}=x+\int_{0}^{t} \vartheta_{u} \cdot d S_{u}, \quad 0 \leq t \leq T .
$$

In order to avoid doubling strategies, we restrict as usual to strategies leading to bounded from below wealth processes. We denote by $\mathfrak{X}^{b}(x)$ the family of terminal values of wealth processes starting from $x$ such that the above holds:

$$
\mathfrak{X}^{b}(x):=\left\{X_{T}^{x, \vartheta}: \vartheta \in \Theta, X^{x, \vartheta} \geq-\kappa \text { on }[0, T] \text { for some } \kappa \in \mathbb{R}\right\} .
$$

Note that $\mathfrak{X}^{b}(x)=x+\mathfrak{X}^{b}(0)$.

As usual, a probability measure $\mathbb{Q}$ is called an equivalent local martingale measure if it is equivalent to $\mathbb{P}$ and if $S$ is a $(\mathbb{F}, \mathbb{Q})$-local martingale. The family of equivalent local martingales will be denoted by $\mathcal{M}$. We assume throughout this paper that

$$
\mathcal{M} \neq \emptyset
$$

which ensures the absence of arbitrage opportunities (in the no-free lunch with vanishing risk sense), see [10] for details. In the following, we will often use the notation $\mathbb{Q}^{*}$ to denote a fixed element of $\mathcal{M}$.

Note that we do not impose that $\mathcal{F}_{T}$ equals $\mathcal{F}_{T}^{S}$, where $\mathbb{F}^{S}=\left(\mathcal{F}_{t}^{S}\right)_{t \leq T}$ is the completion of the right-continuous filtration generated by $S$, in order to allow for additional randomness.

However, we shall often consider that the pure financial market is complete in the following sense.

Definition 2.1. We say that the pure financial market is complete, in short (HCM) holds, if

$$
\mathbb{E}^{\mathbb{Q}^{*}}[\xi]=\mathbb{E}^{\mathbb{Q}}[\xi] \text { for all } \mathbb{Q} \in \mathcal{M} \text { and } \xi \in L^{\infty}\left(\mathcal{F}_{T}^{S}\right),
$$

where $L^{\infty}\left(\mathcal{F}_{T}^{S}\right)$ denotes the set of essentially bounded $\mathcal{F}_{T}^{S}$-measurable random variables.

Remark 2.1. Under (HCM), we must have $\xi \in \mathfrak{X}^{b}\left(\mathbb{E}^{\mathbb{Q}}[\xi]\right)$ for all $\xi \in L^{\infty}\left(\mathcal{F}_{T}^{S}\right)$. See [10].

Remark 2.2. Note that, if $\mathcal{F}_{T}^{S} \subsetneq \mathcal{F}_{T}$, then (HCM) only implies that the pure financial market is complete in the sense of Remark 2.1, and not that $\mathcal{M}$ is reduced to a singleton. As an example, assume that we can find $A \in \mathcal{F}_{T}$ such that $\mathbb{P}[A]>0$ and $A$ is independent of $\mathcal{F}_{T}^{S}$ given $\mathcal{F}_{s}$ for all $s \in[0, T]$ under $\mathbb{P}$. Set $H^{*}:=d \mathbb{Q}^{*} / d \mathbb{P}, H^{*}(S):=\mathbb{E}\left[H^{*} \mid \mathcal{F}_{T}^{S}\right]$ and $H_{A}^{\varepsilon}:=\varepsilon H^{*}+(1-\varepsilon) H^{*}(S) \mathbb{1}_{A} / \mathbb{P}[A]$ for some $\varepsilon \in(0,1]$. Then, for any increasing sequence of 
$\mathbb{F}^{S}$-stopping times $\left(\tau_{k}\right)_{k \geq 1}$ such that $S^{(k)}:=S_{\cdot \wedge \tau_{k}}$ is bounded on $[0, T]$ for all $k \geq 1$, we have

$$
\begin{aligned}
\mathbb{E}\left[H_{A}^{\varepsilon} S_{t}^{(k)} \mid \mathcal{F}_{s}\right] & =\varepsilon \mathbb{E}\left[H^{*} \mid \mathcal{F}_{s}\right] S_{s}^{(k)}+(1-\varepsilon) \mathbb{E}\left[\mathbb{E}\left[H^{*} S_{t}^{(k)} \mid \mathcal{F}_{T}^{S}\right] \mathbb{1}_{A} \mid \mathcal{F}_{s}\right] / \mathbb{P}[A] \\
& =\varepsilon \mathbb{E}\left[H^{*} \mid \mathcal{F}_{s}\right] S_{s}^{(k)}+(1-\varepsilon) \mathbb{E}\left[\mathbb{E}\left[H^{*} S_{t}^{(k)} \mid \mathcal{F}_{T}^{S}\right] \mid \mathcal{F}_{s}\right] \mathbb{E}\left[\mathbb{1}_{A} \mid \mathcal{F}_{s}\right] / \mathbb{P}[A] \\
& =\left(\varepsilon \mathbb{E}\left[H^{*} \mid \mathcal{F}_{s}\right]+(1-\varepsilon) \mathbb{E}\left[H^{*}(S) \mid \mathcal{F}_{s}\right] \mathbb{P}\left[A \mid \mathcal{F}_{s}\right] / \mathbb{P}[A]\right) S_{s}^{(k)} \\
& =\mathbb{E}\left[H_{A}^{\varepsilon} \mid \mathcal{F}_{s}\right] S_{s}^{(k)}
\end{aligned}
$$

for $0 \leq s \leq t \leq T$, which shows that the measure $\mathbb{Q}_{A}^{\varepsilon}$ defined by $d \mathbb{Q}_{A}^{\varepsilon} / d \mathbb{P}=H_{A}^{\varepsilon}$ belongs to $\mathcal{M}$. In general $\mathbb{Q}_{A}^{\varepsilon} \neq \mathbb{Q}^{*}$.

Remark 2.3. The same arguments as in Remark 2.2 imply that $\mathbb{Q}^{*}(S)$ defined by $d \mathbb{Q}^{*}(S)=$ $\mathbb{E}\left[d \mathbb{Q}^{*} / d \mathbb{P} \mid \mathcal{F}_{T}^{S}\right] d \mathbb{P}$ belongs to $\mathcal{M}$. Note for later use that $d \mathbb{Q}^{*}(S)=\mathbb{E}\left[d \mathbb{Q} / d \mathbb{P} \mid \mathcal{F}_{T}^{S}\right] d \mathbb{P}$ for any $\mathbb{Q} \in \mathcal{M}$ when (HCM) holds.

Remark 2.4. Assume that $\mathbb{F}$ can be written as $\left(\mathcal{F}_{t}^{S} \vee \mathcal{F}_{t}^{\perp}\right)_{t \leq T}$ for some filtration $\mathbb{F}^{\perp}=\left(\mathcal{F}_{t}^{\perp}\right)_{t \leq T}$ independent of $\mathbb{F}^{S}$ under $\mathbb{P}$, and satisfying the usual conditions. Then, any $A \in \mathcal{F}_{T}^{\perp}$ is independent of $\mathcal{F}_{T}^{S}$ given $\mathcal{F}_{s}$ for all $s \in[0, T]$ under $\mathbb{P}$. Indeed, under the above assumption, $\mathcal{F}_{s}$ is generated by elements of the form $B_{s}^{S} \cap B_{s}^{\perp}$ with $B_{s}^{S} \in \mathcal{F}_{s}^{S}$ and $B_{s}^{\perp} \in \mathcal{F}_{s}^{\perp}$. Given $\xi \in L^{\infty}\left(\mathcal{F}_{T}^{S}\right)$, we then have

$$
\mathbb{E}\left[\xi \mathbb{1}_{B_{s}^{S} \cap B_{s}^{\perp}}\right]=\mathbb{E}\left[\mathbb{E}\left[\xi \mid \mathcal{F}_{s}^{S}\right] \mathbb{1}_{B_{s}^{S}}\right] \mathbb{E}\left[\mathbb{1}_{B_{s}^{\perp}}\right]=\mathbb{E}\left[\mathbb{E}\left[\xi \mid \mathcal{F}_{s}^{S}\right] \mathbb{1}_{B_{s}^{S} \cap B_{s}^{\perp}}\right],
$$

so that $\mathbb{E}\left[\xi \mid \mathcal{F}_{s}^{S}\right]=\mathbb{E}\left[\xi \mid \mathcal{F}_{s}\right]$. Similarly, $\mathbb{E}\left[\mathbb{1}_{A} \mid \mathcal{F}_{s}^{\perp}\right]=\mathbb{E}\left[\mathbb{1}_{A} \mid \mathcal{F}_{s}\right]$. Moreover,

$$
\mathbb{E}\left[\xi \mathbb{1}_{A} \mathbb{1}_{B_{s}^{S} \cap B_{s}^{\perp}}\right]=\mathbb{E}\left[\xi \mathbb{1}_{B_{s}^{S}}\right] \mathbb{E}\left[\mathbb{1}_{A} \mathbb{1}_{B_{s}^{\perp}}\right]=\mathbb{E}\left[\mathbb{E}\left[\xi \mid \mathcal{F}_{s}^{S}\right] \mathbb{1}_{B_{s}^{S}}\right] \mathbb{E}\left[\mathbb{E}\left[\mathbb{1}_{A} \mid \mathcal{F}_{s}^{\perp}\right] \mathbb{1}_{B_{s}^{\perp}}\right]
$$

which, combined with the above assertions, leads to

$$
\mathbb{E}\left[\xi \mathbb{1}_{A} \mathbb{1}_{B_{s}^{S} \cap B_{s}^{\perp}}\right]=\mathbb{E}\left[\mathbb{E}\left[\xi \mid \mathcal{F}_{s}^{S}\right] \mathbb{1}_{B_{s}^{S}} \mathbb{E}\left[\mathbb{1}_{A} \mid \mathcal{F}_{s}^{\perp}\right] \mathbb{1}_{B_{s}^{\perp}}\right]=\mathbb{E}\left[\mathbb{E}\left[\xi \mid \mathcal{F}_{s}\right] \mathbb{E}\left[\mathbb{1}_{A} \mid \mathcal{F}_{s}\right] \mathbb{1}_{B_{s}^{S} \cap B_{s}^{\perp}}\right]
$$

Hence, $\mathbb{E}\left[\xi \mathbb{1}_{A} \mid \mathcal{F}_{s}\right]=\mathbb{E}\left[\xi \mid \mathcal{F}_{s}\right] \mathbb{E}\left[\mathbb{1}_{A} \mid \mathcal{F}_{s}\right]$.

\subsection{Diversification and utility based pricing}

We are interested in the pricing by utility indifference, see [13], of aggregated claims of the form

$$
G_{n}:=\sum_{i=1}^{n} g^{i}, n \geq 1,
$$

where $\left(g^{i}\right)_{i \geq 1}$ is a given sequence of random variables.

Although the specific structure of the $G_{n}$ 's is not so important from the mathematical point of view, we have in mind that each $g^{i}$ corresponds to a contingent claim sold by an insurance company to a specific agent $i$, and that the $g^{i}$ s have the same law and are independent conditionally to $\mathcal{F}_{T}^{S}$ under $\mathbb{P}$. This means that the $g^{i}$ 's may depend of two sources or risks. One related to the pure financial market behavior, i.e. $S$, the other one coming from an external source of randomness which only depends on the agent $i$.

Example 2.1 (Revenue insurance). Let $S^{1}$ denote the spot price of one quintal of wheat on the financial market and let $Y^{i}$ denotes the number of quintals produced by the farmer $i$ at time $T$. The payoff of a revenue insurance takes the form $g^{i}=\left[K-Y^{i} S_{T}\right]^{+}$for some strike $K>0$ fixed in advance. It compensates the losses incurred by the farmer $i$ if his revenue $Y^{i} S_{T}$, induced by the sale of the production at the spot price $S_{T}$ at time $T$, is less than a targeted level $K$. If the wheat market contains enough futures and provides enough liquidity, we can consider that it is complete. Moreover, we can also consider that the global level of production (at the level of a sufficiently large area) is already reflected into the prices so that the $Y^{i}$ 's can be assumed to be independent given $\mathcal{F}_{T}^{S}$. 
Example 2.2 (Mortality derivatives). A simple example takes the form $g^{i}=f\left(S, \zeta^{i}\right)$ where $f$ is a real valued measurable map on $\mathbb{D} \times([0, T] \cup\{\infty\})$, with $\mathbb{D}$ denoting the set of càdlàg $\mathbb{R}^{d}$-valued functions on $[0, T]$ (endowed with the Skorohod topology), and $\zeta^{i}$ is a $[0, T]$-valued random variable denoting the time of death of $i$ if it is before $T$ and taking the value $\infty$ otherwise. Again, one source of randomness comes from the financial market, while the $\zeta^{i}$ 's can generally be assumed to be independent and with the same law (at least among a given sub-population).

Under the above interpretation, the global liability of the insurance company is $G_{n}$ if the contracts have been subscribed by the clients 1 to $n$. If the insurance company does not differentiate her clients, then she has to fix the same price $p_{n}$ to each of them.

If the global market was complete, meaning that $\mathcal{M}=\left\{\mathbb{Q}^{*}\right\}$, and the law of $G_{n} / n$ under $\mathbb{Q}^{*}$ was independent on $n$, then $p_{n}$ should be equal to $\bar{p}:=\mathbb{E}^{\mathbb{Q}^{*}}\left[G_{n} / n\right]$. Obviously the completeness of the global market typically fails for the examples we have in mind. Still, as explained in the introduction, this pricing rule has been proposed in the literature for the case where (HCM) holds and $\left(g^{i}\right)_{i \geq 1}$ is a sequence of independent and identically distributed random variables given $\mathcal{F}_{T}^{S}$. The later has essentially to be understood in the sense that one can appeal to the law of large numbers, at least conditionally to $\mathcal{F}_{T}^{S}$, so that

$$
G_{n} / n \rightarrow \bar{g} \mathbb{P}-\text { a.s. for some } \bar{g} \in L^{\infty}\left(\mathcal{F}_{T}^{S}\right) \text {. }
$$

Under (HCM), one can indeed find $\vartheta \in \Theta$ such that

$$
X_{T}^{\bar{p}, \vartheta}=\bar{g} \text { for } \bar{p}=\mathbb{E}^{\mathbb{Q}^{*}}[\bar{g}],
$$

recall Remark 2.1. Then, (2.3) implies that

$$
\left(X_{T}^{n \bar{p}, n \vartheta}-G_{n}\right) / n \rightarrow 0 \quad \mathbb{P}-\text { a.s. }
$$

i.e. by replicating $n \bar{g}$ we end up with a hedging error that converges $\mathbb{P}-$ a.s. to 0 . This is achieved by considering the strategy $n \vartheta$ and starting from the $n$ initial premiums, each equal to $\bar{p}$.

This is however inconsistent with the typical behavior of a risk adverse agent. In particular, it has no reason to be in accordance with a (unit) utility indifference price defined by

$$
p_{n}\left(G_{n}, U\right):=\inf \left\{p \in \mathbb{R}: \sup _{X \in \mathfrak{X}^{b}(n p)} \mathbb{E}\left[U\left(X-G_{n}\right)\right] \geq \sup _{X \in \mathfrak{X}^{b}(0)} \mathbb{E}[U(X)]\right\},
$$

where $U$ is a concave non-decreasing function viewed as a utility function, and where we restrict to a 0 initial endowment (before selling the claims) without loss of generality since any fixed initial wealth can be incorporated in the utility function by a simple translation argument.

We conclude this section with simple counter-examples. The first one concerns utility functions with bounded from below domains.

Example 2.3 (Utility with bounded from below domain). Let $U$ be concave non-decreasing with values in $\mathbb{R} \cup\{-\infty\}$ such that $|U(0)|+|U(\infty)|<\infty$ and $U(-c)=-\infty$ for some $c>0$. Let $p_{n}:=p_{n}\left(G_{n}, U\right)$ be defined as in (2.4). Since $U(0)>-\infty$ and $U$ is bounded from above, for each $\varepsilon>0$, there must exist $\xi^{n, \varepsilon} \in \mathfrak{X}^{b}(0)$ such that $n p_{n}+\varepsilon+\xi^{n, \varepsilon} \geq G_{n}-c$. This implies that $p_{n} \geq \sup _{\mathbb{Q} \in \mathcal{M}} \mathbb{E}^{\mathbb{Q}}\left[G_{n} / n\right]-c / n$ and therefore

$$
\liminf _{n \rightarrow \infty} p_{n} \geq \liminf _{n \rightarrow \infty} \sup _{\mathbb{Q} \in \mathcal{M}} \mathbb{E}^{\mathbb{Q}}\left[G_{n} / n\right] .
$$

Let us now concentrate on the case where $\left(g^{i}\right)_{i \geq 1}$ is defined as in Example 2.2 with $f$ bounded. Assume that the $\zeta^{i}$ 's form an independent family with a common law under $\mathbb{P}$, and that $\sigma\left(\zeta^{i}, i \geq\right.$ 1) $\subset \mathcal{F}_{T}^{\perp}$ with $\mathbb{F}^{\perp}$ satisfying the conditions of Remark 2.4. Note that

$$
\mathbb{E}\left[H^{*}(S) f\left(S, \zeta^{i}\right) \mid \mathcal{F}_{T}^{\perp}\right]=\mathbb{E}\left[H^{*}(S) f\left(S, \zeta^{i}\right) \mid \zeta^{i}\right], \quad i \geq 1,
$$


where $H^{*}(S)$ is defined as in Remark 2.2. Set $\psi:=\operatorname{esssup} \mathbb{E}\left[H^{*}(S) f\left(S, \zeta^{1}\right) \mid \zeta^{1}\right]$. For $k, n \geq 1$ and $\varepsilon \in(0,1)$, we define $A_{k}^{n}:=\left\{\mathbb{E}\left[H^{*}(S) f\left(S, \zeta^{i}\right) \mid \zeta^{i}\right] \geq \psi-k^{-1}\right.$, for all $\left.i \leq n\right\}$ and $H_{n, k}^{\varepsilon}:=$ $\varepsilon H^{*}+(1-\varepsilon) H^{*}(S) \mathbb{1}_{A_{k}^{n}} / \mathbb{P}\left[A_{k}^{n}\right]$. Note that $\mathbb{P}\left[A_{k}^{n}\right]>0$ since the $\zeta^{i}$ 's are independent and have the same law under $\mathbb{P}$. Then, according to Remark 2.4 and Remark 2.2, $\mathbb{Q}_{n, k}^{\varepsilon}:=H_{n, k}^{\varepsilon} \cdot \mathbb{P} \in \mathcal{M}$. Recalling (2.5), this implies that

$$
\liminf _{n \rightarrow \infty} p_{n} \geq \liminf _{n \rightarrow \infty} \lim _{k \rightarrow 0} \lim _{\varepsilon \rightarrow 0} \mathbb{E}^{\mathbb{Q}_{n, k}^{\varepsilon}}\left[G_{n} / n\right]=\psi .
$$

Clearly, the above lower bound is typically strictly larger than $\mathbb{E}^{\mathbb{Q}^{*}}\left[\mathbb{E}\left[f\left(S, \zeta^{1}\right) \mid \mathcal{F}_{T}^{S}\right]\right]$, while applying the law of large numbers conditionally to $\mathcal{F}_{T}^{S}$ implies that $G_{n} / n \rightarrow \bar{g}=\mathbb{E}\left[f\left(S, \zeta^{1}\right) \mid \mathcal{F}_{T}^{S}\right]$ $\mathbb{P}$ - a.s. For instance, if $f$ is lower-semicontinuous, non-decreasing with respect to its second parameter, and if each $\zeta^{i}$ has a support equal to $\left[y_{\min }, y_{\max }\right] \subset \mathbb{R}$ under $\mathbb{P}$, then $\psi=$ $\mathbb{E}\left[H^{*}(S) f\left(S, y_{\max }\right)\right]=\mathbb{E}^{\mathbb{Q}^{*}}\left[f\left(S, y_{\max }\right)\right]=\mathbb{E}^{\mathbb{Q}^{*}}\left[\max \left\{f(S, y), y_{\min } \leq y \leq y_{\max }\right\}\right]$. Under $(\mathbf{H C M})$, the later is the hedging price of $\max \left\{f(S, y), y_{\min } \leq y \leq y_{\max }\right\}$, recall Remark 2.1, so that $p_{n} \leq \psi$ for all $n \geq 1$, and therefore $p_{n} \rightarrow \psi$.

In general, going through utility function with unbounded domain does not help, as show in our second example.

Example 2.4 (Exponential utility function). Let $U$ be an exponential utility function of the form $U^{\eta}(y)=-e^{-\eta y}, \eta>0$. Assume that the $g^{i}$ 's have the form taken in Example 2.3, that $\mathcal{F}_{t}^{\perp}=\sigma\left(\mathcal{F}_{t}^{\perp, i}, i \geq 1\right), t \leq T$, for some filtrations $\left(\mathbb{F}^{\perp, i}\right)_{i \geq 1}$ such that $\mathcal{F}_{T}^{S}, \mathcal{F}_{T}^{\perp, 1}, \mathcal{F}_{T}^{\perp, 2}, \ldots$ are independent under $\mathbb{P}$, and that $\zeta^{i}$ is $\mathcal{F}_{T}^{\perp, i}$-measurable for each $i \geq 1$. Then, it can be shown that $p_{n}^{\eta}:=p_{n}\left(G_{n}, U^{\eta}\right)=p_{1}\left(g^{1}, U^{\eta}\right)=: p^{\eta}$ whenever there exists a unique element of $\mathcal{M}$ with finite relative entropy and whose density is $\mathcal{F}_{T}^{S}$-measurable, see Theorem 4.10 in [2]. It is in particular the case if $(\mathbf{H C M})$ holds and $\mathbb{Q}^{*}(S)$ defined in Remark 2.3 has a finite relative entropy. On the other hand, it is well-known that $p^{\eta}$ converges to the super-hedging price of $g^{1}$ as $\eta \rightarrow \infty$, see [9] and [2]. This implies that, for all $\varepsilon>0$, we can find $\eta_{\varepsilon}$ large enough so that $\lim _{n} p_{n}^{\eta_{\varepsilon}}=p^{\eta_{\varepsilon}} \geq \sup _{\mathbb{Q} \in \mathcal{M}} \mathbb{E}^{\mathbb{Q}}\left[g^{1}\right]-\varepsilon$. For $\varepsilon>0$ small enough, this is again typically greater than $\mathbb{E}^{\mathbb{Q}^{*}}\left[\mathbb{E}\left[f\left(S, \zeta^{1}\right) \mid \mathcal{F}_{T}^{S}\right]\right]$.

\section{Asymptotic diversification rule}

As pointed out in the previous examples, the convergence of the mean aggregated claim $G_{n} / n$ to a replicable claim $\bar{g} \in L^{\infty}\left(\mathcal{F}_{T}^{S}\right)$ is not enough in order to ensure the convergence of its unit utility indifference price $p_{n}\left(G_{n}, U\right)$ to $\mathbb{E}^{\mathbb{Q}^{*}}[\bar{g}]$. Intuitively, this can be recovered only if the risk aversion vanishes as the number of sold claims goes to infinity.

Hence, we suppose from now on that the preferences of the agent are characterized by a sequence of utility functions $\left(U_{n}\right)_{n \geq 1}$, which depends on the number $n$ of sold claims $\left(g^{i}\right)_{0<i<n}$. The purpose of this section is to investigate the asymptotic behavior of the corresponding unit utility indifference price when $n$ times the absolute risk aversion of $U_{n}$ vanishes to 0 as $n$ goes to $\infty$.

We first provide a general characterization of the asymptotic unit utility indifference price in terms of the sequence of associated dual pricing measures. Whenever the pure financial market is complete, i.e. (HCM) is satisfied, this limit identifies to the risk neutral price of $\bar{g}$. When the pure financial market is incomplete, it does not seem possible to obtain a precise characterization of the limit price, except when $\left(U_{n}\right)_{n \geq 1}$ behaves asymptotically like a sequence of exponential utility functions with vanishing absolute risk aversion. In this case, we prove that the asymptotic price coincides with the price of $\bar{g}$ under the minimal entropy martingale measure.

\subsection{General convergence result}

In this section, we consider a sequence of twice continuously differentiable, strictly concave and increasing utility functions $\left(U_{n}\right)_{n \geq 1}$ defined on the whole real line and satisfying the Inada conditions:

$$
U_{n}^{\prime}(-\infty)=\lim _{x \rightarrow-\infty} U_{n}^{\prime}(x)=\infty \quad \text { and } \quad U_{n}^{\prime}(\infty)=\lim _{x \rightarrow \infty} U_{n}^{\prime}(x)=0, \quad n \geq 1 .
$$


Besides, we suppose that all the utility functions have a reasonable asymptotic elasticity as defined in [19], i.e.

$$
\limsup _{x \rightarrow \infty} \frac{x U_{n}^{\prime}(x)}{U_{n}(x)}<1, \quad \liminf _{x \rightarrow-\infty} \frac{x U_{n}^{\prime}(x)}{U_{n}(x)}>1, \quad n \geq 1 .
$$

We finally introduce the convex conjugates of the $U_{n}$ 's defined by

$$
V_{n}: y \in(0, \infty) \mapsto \sup _{x \in \mathbb{R}}\left\{U_{n}(x)-x y\right\},
$$

and assume that the dual problems are finite:

$$
\left\{(y, \mathbb{Q}) \in(0, \infty) \times \mathcal{M}: \mathbb{E}\left[V_{n}(y d \mathbb{Q} / d \mathbb{P})\right]<\infty\right\} \neq \emptyset \text { for all } n \geq 1
$$

Under the additional uniform boundedness assumption

$$
\sup _{n \geq 1}\left\|G_{n} / n\right\|_{L^{\infty}}<\infty
$$

the unit utility indifference prices $p_{n}\left(G_{n}, U_{n}\right)$ given by (2.4) are well defined for any $n \geq 1$ and existence for the optimal dual probability and multiplier, given by

$$
\left(y_{n}^{0}, \mathbb{Q}_{n}^{0}\right):=\arg \min \left\{\mathbb{E}\left[V_{n}\left(y \frac{d \mathbb{Q}}{d \mathbb{P}}\right)\right],(y, \mathbb{Q}) \in(0, \infty) \times \mathcal{M}\right\},
$$

is guaranteed, see e.g. Theorem 3.1, Remark 3.3 and Proposition 3.1 in [3] (see also [19] for a fomulation in terms of absolutely continuous local martingale measures).

In the rest of this section, we work under the standing assumption:

Assumption 3.1. The conditions (3.1), (3.2), (3.3) and (3.4) hold.

In order to derive the asymptotic behavior of the unit indifference price, we now also suppose that the agent tends to become risk neutral when selling a large number of claims. More precisely, we shall work under the following additional condition on the asymptotic absolute risk aversion:

$$
n\left\|r_{n}\right\|_{\infty} \underset{n \rightarrow \infty}{\longrightarrow} 0, \quad \text { with } \quad r_{n}: x \mapsto-\frac{U_{n}^{\prime \prime}(x)}{U_{n}^{\prime}(x)},
$$

and $\left\|r_{n}\right\|_{\infty}:=\sup _{x \in \mathbb{R}}\left|r_{n}(x)\right|$.

Theorem 3.1. Let Assumption 3.1 and (3.6) hold. Then, the sequence of utility indifference prices satisfies

$$
\liminf _{n \rightarrow \infty} p_{n}\left(G_{n}, U_{n}\right)=\liminf _{n \rightarrow \infty} \mathbb{E}^{\mathbb{Q}_{n}^{0}}\left[G_{n} / n\right] \text { and } \limsup _{n \rightarrow \infty} p_{n}\left(G_{n}, U_{n}\right)=\limsup _{n \rightarrow \infty} \mathbb{E}^{\mathbb{Q}_{n}^{0}}\left[G_{n} / n\right] .
$$

Proof. We set $p_{n}:=p_{n}\left(G_{n}, U_{n}\right)$ for ease of notations. We only provide the proof for the lim inf, the other one being similar.

1. Given $n \geq 1$, it follows from standard duality arguments, see [16] and [3], that

$$
\inf _{y>0, \mathbb{Q} \in \mathcal{M}} \mathbb{E}\left[V_{n}\left(y \frac{d \mathbb{Q}}{d \mathbb{P}}\right)+y n p_{n}-y \frac{d \mathbb{Q}}{d \mathbb{P}} G_{n}\right]=\inf _{y>0, \mathbb{Q} \in \mathcal{M}} \mathbb{E}\left[V_{n}\left(y \frac{d \mathbb{Q}}{d \mathbb{P}}\right)\right]=\mathbb{E}\left[V_{n}\left(y_{n}^{0} \frac{d \mathbb{Q}_{n}^{0}}{d \mathbb{P}}\right)\right],
$$

recall (3.5). Taking in particular $(y, \mathbb{Q})=\left(y_{n}^{0}, \mathbb{Q}_{n}^{0}\right)$, this implies that

$$
\mathbb{E}\left[V_{n}\left(y_{n}^{0} \frac{d \mathbb{Q}_{n}^{0}}{d \mathbb{P}}\right)+y_{n}^{0} n p_{n}-y_{n}^{0} \frac{d \mathbb{Q}_{n}^{0}}{d \mathbb{P}} G_{n}\right] \geq \mathbb{E}\left[V_{n}\left(y_{n}^{0} \frac{d \mathbb{Q}_{n}^{0}}{d \mathbb{P}}\right)\right],
$$

and therefore $p_{n} \geq \mathbb{E}^{\mathbb{Q}_{n}^{0}}\left[G_{n} / n\right]$.

2. On the other hand, it follows from [19] that, for $n \geq 1$, we can find $\hat{X}^{n} \in L^{0}\left(\mathcal{F}_{T}\right)$ such that

$$
\sup _{X \in \mathfrak{X}^{b}(0)} \mathbb{E}\left[U_{n}\left(X_{T}\right)\right]=\mathbb{E}\left[U_{n}\left(\hat{X}^{n}\right)\right]
$$


and for which there exists a sequence of optimizers $\left(X^{k, n}\right)_{k \geq 1} \subset \mathfrak{X}^{b}(0)$ such that

$$
U_{n}\left(X_{T}^{n, k}\right) \underset{k \rightarrow \infty}{\stackrel{L^{1}(\mathbb{P})}{\longrightarrow}} U_{n}\left(\hat{X}^{n}\right) .
$$

In order to upper-bound $p_{n}$, we then introduce the following candidate

$$
\pi_{n}:=\inf \left\{p \in \mathbb{R}: \mathbb{E}\left[U_{n}\left(n p+\hat{X}^{n}-G_{n}\right)\right] \geq \mathbb{E}\left[U_{n}\left(\hat{X}^{n}\right)\right]\right\} .
$$

a. We first check that

$$
\pi_{n} \geq p_{n}, \text { for all } n \geq 1 .
$$

To this purpose, it suffices to fix $n \in \mathbb{N}$ and show that

$$
U_{n}\left(n p+X_{T}^{n, k}-G_{n}\right) \underset{k \rightarrow \infty}{\stackrel{L^{1}(\mathbb{P})}{\rightarrow}} U_{n}\left(n p+\hat{X}^{n}-G_{n}\right) \in L^{1}(\mathbb{P}), \text { for all } p \in \mathbb{R} .
$$

To see that the above holds, first note that

$$
\left|U_{n}\left(n p+X_{T}^{n, k}-G_{n}\right)-U_{n}\left(n p+\hat{X}^{n}-G_{n}\right)\right|=\left|\int_{\hat{X}^{n}}^{X_{T}^{n, k}} U_{n}^{\prime}\left(n p+t-G_{n}\right) d t\right| .
$$

Consider now the relation

$$
\log U_{n}^{\prime}\left(n p+t-G_{n}\right)-\log U_{n}^{\prime}(t) \leq n\left\|\frac{U_{n}^{\prime \prime}}{U_{n}^{\prime}}\right\|_{\infty}\left|p-\frac{G_{n}}{n}\right|, \quad t, p \in \mathbb{R},
$$

which, together with (3.4) and (3.6) leads to the existence of a constant $C_{p}$ (which only depends on $p$ ) such that

$$
U_{n}^{\prime}\left(n p+t-G_{n}\right) \leq C_{p} U_{n}^{\prime}(t), \text { for all } t \in \mathbb{R} .
$$

Plugging (3.11) into (3.10) gives

$$
\left|U_{n}\left(n p+X_{T}^{n, k}-G_{n}\right)-U_{n}\left(n p+\hat{X}^{n}-G_{n}\right)\right| \leq C_{p}\left|U_{n}\left(X_{T}^{n, k}\right)-U_{n}\left(\hat{X}^{n}\right)\right| .
$$

Hence, (3.9) follows from (3.7), which proves (3.8).

b. We now conclude the proof by providing a lower bound for $\liminf _{n \rightarrow \infty} \pi_{n}$. By definition of $\pi_{n}$, the continuity of the non-increasing function $U_{n},(3.9)$ and the monotone convergence theorem, we have

$$
\mathbb{E}\left[U_{n}\left(n \pi_{n}+\hat{X}^{n}-G_{n}\right)\right]=\mathbb{E}\left[U_{n}\left(\hat{X}^{n}\right)\right] .
$$

This implies that

$$
\mathbb{E}\left[U_{n}\left(\hat{X}^{n}\right)\right]=\mathbb{E}\left[U_{n}\left(\hat{X}^{n}\right)+U_{n}^{\prime}\left(\hat{X}^{n}\right)\left(n \pi_{n}-G_{n}\right)+\frac{1}{2} U_{n}^{\prime \prime}\left(\xi_{n}\right)\left(n \pi_{n}-G_{n}\right)^{2}\right],
$$

where $\xi_{n}$ is a random variable lying in the (random) interval $I_{n}$ formed by $\hat{X}^{n}$ and $n \pi_{n}+\hat{X}^{n}-G_{n}$. We now use the fact that $U_{n}^{\prime}\left(\hat{X}^{n}\right)=y_{n}^{0} \frac{d \mathbb{Q}_{n}^{0}}{d \mathbb{P}}$, recall (3.5) and see [19] and [3], to deduce that

$$
\mathbb{E}^{\mathbb{Q}_{n}^{0}}\left[\pi_{n}-\frac{G_{n}}{n}-\frac{n r_{n}\left(\xi_{n}\right)}{2} \frac{U_{n}^{\prime}\left(\xi_{n}\right)}{U_{n}^{\prime}\left(\hat{X}^{n}\right)}\left(\pi_{n}-\frac{G_{n}}{n}\right)^{2}\right]=0 .
$$

We shall prove in c. below that

$$
\sup _{n \geq 1}\left|\frac{U_{n}^{\prime}\left(\xi_{n}\right)}{U_{n}^{\prime}\left(\hat{X}^{n}\right)}\left(\pi_{n}-\frac{G_{n}}{n}\right)^{2}\right|<\infty .
$$

Combining this last estimate with (3.6) and (3.13) leads to

$$
\liminf _{n \rightarrow \infty} \pi_{n} \leq \liminf _{n \rightarrow \infty} \mathbb{E}^{\mathbb{Q}_{n}^{0}}\left[G_{n} / n\right]
$$


which together with (3.8) and step 1. concludes the proof.

c. It remains to prove the claim (3.14). To see that it holds, we first appeal to (3.12) to deduce that $\mathbb{E}\left[U_{n}^{\prime}\left(\tilde{\xi}_{n}\right)\left(n \pi_{n}-G_{n}\right)\right]=0$ for some random variable $\tilde{\xi}_{n}$ such that $\tilde{\xi}_{n} \in I_{n}$. Since $U_{n}$ is strictly increasing, we deduce from (3.4) that

$$
\left|\pi_{n}-G_{n} / n\right|=\frac{\mathbb{E}\left[U_{n}^{\prime}\left(\tilde{\xi}_{n}\right)\left|G_{n}\right| / n\right]}{\mathbb{E}\left[U_{n}^{\prime}\left(\tilde{\xi}_{n}\right)\right]}+\left|G_{n} / n\right| \leq C \frac{\mathbb{E}\left[U_{n}^{\prime}\left(\tilde{\xi}_{n}\right)\right]}{\mathbb{E}\left[U_{n}^{\prime}\left(\tilde{\xi}_{n}\right)\right]}+C=2 C, \quad n \geq 1,
$$

for some constant $C>0$. Similarly, since $\xi_{n} \in I_{n}$, we have

$$
\log \left(U_{n}^{\prime}\left(\xi_{n}\right)\right)-\log \left(U_{n}^{\prime}\left(\hat{X}^{n}\right)\right) \leq\left\|\frac{U_{n}^{\prime \prime}}{U_{n}^{\prime}}\right\|_{\infty}\left|\xi_{n}-\hat{X}^{n}\right| \leq n\left\|\frac{U_{n}^{\prime \prime}}{U_{n}^{\prime}}\right\|_{\infty}\left|\pi_{n}-\frac{G_{n}}{n}\right|, \quad n \geq 1,
$$

which is bounded uniformly in $n$ thanks to (3.6) and (3.15).

Remark 3.1. Let $\left(p_{\varphi(n)}\right)_{n \geq 1}$ be a convergent subsequence of $\left(p_{n}\left(G_{n}, U_{n}\right)\right)_{n \geq 1}$. Then, the same arguments as above show that $\lim _{n \rightarrow \infty} p_{\varphi(n)}=\lim _{n \rightarrow \infty} \mathbb{E}^{\mathbb{Q}_{\varphi(n)}^{0}}\left[G_{\varphi(n)} / \varphi(n)\right]$ whenever $\varphi(n) r_{\varphi(n)} \rightarrow 0$.

Remark 3.2. Note that a similar result can be obtained for the indifference price of the aggregated risk $p_{1}\left(G_{n}, U_{n}\right)$ under the weaker condition $\left\|r_{n}\right\|_{\infty} \rightarrow 0$, whenever $\left(G_{n}\right)_{n \geq 1}$ is assumed to be uniformly bounded in $L^{\infty}$. Indeed, a straightforward adaptation of the above proof shows that, under the above conditions,

$$
\liminf _{n \rightarrow \infty} p_{1}\left(G_{n}, U_{n}\right)=\liminf _{n \rightarrow \infty} \mathbb{E}^{\mathbb{Q}_{n}^{0}}\left[G_{n}\right] \text { and } \limsup _{n \rightarrow \infty} p_{1}\left(G_{n}, U_{n}\right)=\limsup _{n \rightarrow \infty} \mathbb{E}^{\mathbb{Q}_{n}^{0}}\left[G_{n}\right] .
$$

This provides a general convergence result for bounded sequences of contingent claims when the absolute risk aversion vanishes in the sup norm, which is of own interest.

\section{$3.2 \quad$ Semi-complete markets}

The representation of the asymptotic unit utility indifference price presented in Theorem 3.1 does not provide a-priori an exact formulation, except in particular cases. When the pure financial market is complete, i.e. (HCM) is satisfied, and (2.3) holds, we verify hereafter that it coincides with the price under the risk neutral measure $\mathbb{Q}^{*}$ of the replicable claim $\bar{g}$.

Corollary 3.1. Let the conditions of Theorem 3.1 hold. Assume further that (2.3) and (HCM) are satisfied. Then the sequence of unit utility indifference prices satisfies

$$
\lim _{n \rightarrow \infty} p_{n}\left(G_{n}, U_{n}\right)=\mathbb{E}^{\mathbb{Q}^{*}}[\bar{g}] .
$$

Proof. In view of Theorem 3.1, it suffices to show that (HCM) implies that the minimal dual measure $\mathbb{Q}_{n}^{0}$, see $(3.5)$, coincides with $\mathbb{Q}^{*}(S)$ as constructed in Remark 2.3, and to apply the dominated convergence theorem, recall (3.4) and (2.3):

$$
\lim _{n \rightarrow \infty} p_{n}\left(G_{n}, U_{n}\right)=\lim _{n \rightarrow \infty} \mathbb{E}^{\mathbb{Q}_{n}^{0}}\left[G_{n} / n\right]=\lim _{n \rightarrow \infty} \mathbb{E}^{\mathbb{Q}^{*}(S)}\left[G_{n} / n\right]=\mathbb{E}^{\mathbb{Q}^{*}(S)}[\bar{g}]=\mathbb{E}^{\mathbb{Q}^{*}}[\bar{g}]
$$

where the last equality follows from the fact that $\bar{g}$ is $\mathcal{F}_{T}^{S}$-measurable.

To see this, we use the convexity of $V_{n}$ to obtain that $\mathbb{E}\left[V_{n}\left(y_{n}^{0} \frac{d \mathbb{Q}_{n}^{0}}{d \mathbb{P}}\right)\right] \geq \mathbb{E}\left[V_{n}\left(y_{n}^{0} H_{n}^{0}(S)\right)\right]$ where $H_{n}^{0}(S):=\mathbb{E}\left[\frac{d \mathbb{Q}_{n}^{0}}{d \mathbb{P}} \mid \mathcal{F}_{T}^{S}\right]=d \mathbb{Q}^{*}(S) / d \mathbb{P}$ by Remark 2.3 , under $(\mathbf{H C M})$. Since $\mathbb{Q}^{*}(S) \in \mathcal{M}$ by Remark 2.3 again, this proves the claim.

Remark 3.3. If the $g^{i}$ 's have the same law and form an independent family conditionally to $\mathcal{F}_{T}^{S}$ under $\mathbb{P}$, then applying the law of large numbers conditionally to $\mathcal{F}_{T}^{S}$ implies $\bar{g}=\mathbb{E}\left[g^{1} \mid \mathcal{F}_{T}^{S}\right]$, so that $\lim _{n \rightarrow \infty} p_{n}\left(G_{n}, U_{n}\right)=\mathbb{E}^{\mathbb{Q}^{*}}\left[\mathbb{E}\left[g^{1} \mid \mathcal{F}_{T}^{S}\right]\right]$. We thus retrieve asymptotically the hybrid pricing rule which consists in taking the mean on the part of the risk that can be diversified and computing the hedging price of the resulting replicable claim. 
Remark 3.4. In [2], the author refers to semi-complete product models to designate situations where the filtration $\mathbb{F}$ and $\mathcal{M}$ have the structure specified in Example 2.4, in particular:

$\left(\mathbf{H C M}^{\mathrm{e}}\right)$ : there exists only one element $\mathbb{Q}^{\mathrm{e}}(S)$ of $\mathcal{M}$ with finite relative entropy and whose density with respect to $\mathbb{P}$ is $\mathcal{F}_{T}^{S}$-measurable.

This later condition is weaker than (HCM), up to the restriction related to the finite relative entropy. However, if we are only interested in utility functions of exponential type, the same argument as in the proof of Corollary 3.1 above shows that $\mathbb{Q}_{n}^{0}=\mathbb{Q}^{\mathrm{e}}(S)$ whenever $\left(\mathbf{H C M}^{\mathrm{e}}\right)$ holds. Assuming further that the conditions of Theorem 3.1 and (2.3) hold, this leads to $\lim _{n \rightarrow \infty} p_{n}\left(G_{n}, U_{n}\right)=\mathbb{E}^{\mathbb{Q}^{\mathrm{e}}(S)}[\bar{g}]$.

\subsection{Incomplete markets and asymptotically exponential utility behav- iors}

In a general incomplete framework, it seems to be hopeless to interpret the limit of $\mathbb{E}^{\mathbb{Q}_{n}^{0}}\left[G_{n} / n\right]$ as the expectation under some martingale measure of $\bar{g}$, the limit of $G_{n} / n$, except if the $U_{n}$ 's are all of exponential type, compare with Remark 3.4.

In this section, we show that the convergence result of Remark 3.4 remains true even if the utility functions do not have a constant absolute risk aversion but only asymptotically behave like a sequence of exponential utility functions in the following sense.

Assumption 3.2. There exist two sequences of strictly positive numbers $\left(\eta_{n}^{1}\right)_{n \geq 1}$ and $\left(\eta_{n}^{2}\right)_{n \geq 1}$ converging toward 0 such that

$$
\begin{aligned}
& 0<\eta_{n}^{2} \leq r_{n}(x) \leq \eta_{n}^{1} \quad \text { for all } x \in \mathbb{R} \text { and } n \geq 1, \\
& \quad \lim _{n \rightarrow \infty} \eta_{n}^{2} / \eta_{n}^{1}=1 .
\end{aligned}
$$

Remark 3.5. The existence of the sequence $\left(\eta_{n}^{1}\right)_{n \geq 1}$ converging to zero is exactly the content of the assumption (3.6). Assumption 3.2 implies that the function $r_{n}$ is asymptotically bounded in between two sequences converging to zero with the same first order convergence rate. In particular, this assumption includes the case of agents with utility functions of the form $U_{n}$ : $x \mapsto-\left(\lambda_{n}\right)^{-1} e^{-\lambda_{n} x}-\left(\mu_{n}\right)^{-1} e^{-\mu_{n}\left(x+x_{0}\right)}$, for $n \geq 1$ and $x_{0}>0$ as long as the positive sequences $\left(\lambda_{n}\right)_{n \geq 1}$ and $\left(\mu_{n}\right)_{n \geq 1}$ are equivalent as $n$ goes to $\infty$.

It follows from Lemma 3.1 below that (3.3) is equivalent to

$$
\left\{\mathbb{Q} \in \mathcal{M}: \mathbb{E}\left[\frac{d \mathbb{Q}}{d \mathbb{P}} \log \left(\frac{d \mathbb{Q}}{d \mathbb{P}}\right)\right]<\infty\right\} \neq \emptyset
$$

whenever Assumption 3.2 holds. Hence, Assumption 3.1 can now be formulated as

Assumption 3.3. The conditions (3.1), (3.2), (3.18) and (3.4) hold.

In the following, we denote by $\mathbb{Q}^{e}$ the element of $\mathcal{M}$ that minimizes the relative entropy,

$$
\mathbb{E}\left[\frac{d \mathbb{Q}^{e}}{d \mathbb{P}} \log \left(\frac{d \mathbb{Q}^{e}}{d \mathbb{P}}\right)\right]=\inf _{\mathbb{Q} \in \mathcal{M}} \mathbb{E}\left[\frac{d \mathbb{Q}}{d \mathbb{P}} \log \left(\frac{d \mathbb{Q}}{d \mathbb{P}}\right)\right],
$$

and whose existence is guaranteed by Theorem 2.2 in [8].

Remark 3.6. The map $y>0 \mapsto y \ln y$ being strictly convex, it follows from Remark 2.3 that $d \mathbb{Q}^{e} / d \mathbb{P}$ is $\mathcal{F}_{T}^{S}$-measurable, recall the argument used in the proof of Corollary 3.1. However, we do not impose in (3.18) the uniqueness condition of $\left(\mathbf{H C M}^{\mathrm{e}}\right)$ in Remark 3.4.

Theorem 3.2. Let Assumption 3.2, Assumption 3.3 and (2.3) be in force. Then the sequence of unit utility indifference prices satisfies

$$
\lim _{n \rightarrow \infty} p_{n}\left(G_{n}, U_{n}\right)=\mathbb{E}^{\mathbb{Q}^{e}}[\bar{g}] .
$$


Remark 3.7. When the $g^{i}$ 's satisfy the conditions of Remark 3.3, the above result shows that the unit indifference prices converges to $\mathbb{E}^{\mathbb{Q}^{e}}\left[\mathbb{E}\left[g^{1} \mid \mathcal{F}_{T}^{S}\right]\right]$. Again, this consists in taking the mean over the part of the risk that can be diversified and computing the price under the minimal entropy martingale measure of the pure financial remaining claim.

In the rest of this section, we provide the proof of Theorem 3.2. In view of Theorem 3.1, it would suffice to show that

$$
\lim _{n \rightarrow \infty} \mathbb{E}^{\mathbb{Q}_{n}^{0}}\left[G_{n} / n\right]=\mathbb{E}^{\mathbb{Q}^{e}}[\bar{g}],
$$

where we recall that $\mathbb{Q}_{n}^{0}$ is defined in (3.5).

In the following, we actually prove that

$$
\lim _{n \rightarrow \infty} \mathbb{E}^{\mathbb{Q}_{n}^{\alpha}}\left[G_{n} / n\right]=\mathbb{E}^{\mathbb{Q}^{e}}[\bar{g}],
$$

where $\left(y_{n}^{\alpha}, \mathbb{Q}_{n}^{\alpha}\right)$ are defined as $\left(y_{n}^{0}, \mathbb{Q}_{n}^{0}\right)$ in $(3.5)$ but with

$$
U_{n}^{\alpha}: x \mapsto \alpha_{n} \frac{U_{n}(x)-U_{n}(0)}{U_{n}^{\prime}(0)}, \quad n \geq 1
$$

in place of $U_{n}$. In the above, $\left(\alpha_{n}\right)_{n \geq 1}$ is a sequence of positive numbers to be chosen later on, see (3.34) in the proof of Lemma 3.2 below. This trick is inspired from [7] and allows to reduce to the case where

$$
U_{n}^{\alpha}(0)=0 \text { and }\left[U_{n}^{\alpha}\right]^{\prime}(0)=\alpha_{n}, n \geq 1 .
$$

Obviously, since $U_{n}^{\alpha}$ is an affine transformation of the utility function $U_{n}$, we have

$$
p_{n}\left(G_{n}, U_{n}\right)=p_{n}\left(G_{n}, U_{n}^{\alpha}\right) .
$$

Recalling Theorem 3.1, (3.20) is thus sufficient to deduce the result of Theorem 3.2.

We first provide upper and lower bounds for the Fenchel transform $V_{n}^{\alpha}$ of $U_{n}^{\alpha}$ in terms of Fenchel transforms of exponential utility functions with risk aversion $\eta_{n}^{1}$ and $\eta_{n}^{2}$.

Lemma 3.1. Let Assumption 3.2 hold. Then, for each $n \geq 1$,

$$
V_{n}^{1}(y) \leq V_{n}^{\alpha}(y) \leq V_{n}^{2}(y), \quad y \in(0, \infty),
$$

where the functions $V_{n}^{1}$ and $V_{n}^{2}$ are defined by

$$
V_{n}^{i}(y):=\frac{y}{\eta_{n}^{i}} \log \left(\frac{y}{\alpha_{n}}\right)+\frac{\alpha_{n}-y}{\eta_{n}^{i}}, \quad y \in(0, \infty), i=1,2 .
$$

Proof. It follows from the definition of $\left(V_{n}^{i}\right)_{i=1,2}$ and (3.21) that

$$
V_{n}^{\alpha}\left(\alpha_{n}\right)=V_{n}^{i}\left(\alpha_{n}\right)=0 \quad \text { and }\left[V_{n}^{\alpha}\right]^{\prime}\left(\alpha_{n}\right)=\left[V_{n}^{i}\right]^{\prime}\left(\alpha_{n}\right)=0, i=1,2 .
$$

Since $r_{n}=-\left[U_{n}^{\alpha}\right]^{\prime \prime} /\left[U_{n}^{\alpha}\right]^{\prime}$ and $\left[U_{n}^{\alpha}\right]^{\prime \prime} \circ\left(\left[U_{n}^{\alpha}\right]^{\prime}\right)^{-1}=1 /\left[V_{n}^{\alpha}\right]^{\prime \prime}$, we deduce from (3.16) in Assumption 3.2 that

$$
\eta_{n}^{2} \leq \frac{1}{y\left[V_{n}^{\alpha}\right]^{\prime \prime}(y)} \leq \eta_{n}^{1}, \quad y \in(0, \infty) .
$$

Together with the strict convexity of $V_{n}^{\alpha}$, of each $V_{n}^{i}$, and the relation $\eta_{n}^{1}\left[V_{n}^{1}\right]^{\prime \prime}(y)=\eta_{n}^{2}\left[V_{n}^{2}\right]^{\prime \prime}(y)=$ $1 / y$, this shows that

$$
\left[V_{n}^{1}\right]^{\prime \prime} \leq\left[V_{n}^{\alpha}\right]^{\prime \prime} \leq\left[V_{n}^{2}\right]^{\prime \prime} .
$$

We now simply deduce from the right-hand side of (3.24) and (3.25) that

$$
\left[V_{n}^{2}\right]^{\prime} \leq\left[V_{n}^{\alpha}\right]^{\prime} \leq\left[V_{n}^{1}\right]^{\prime} \quad \text { on } \quad\left(0, \alpha_{n}\right] \quad \text { and } \quad\left[V_{n}^{2}\right]^{\prime} \geq\left[V_{n}^{\alpha}\right]^{\prime} \geq\left[V_{n}^{1}\right]^{\prime} \quad \text { on } \quad\left[\alpha_{n}, \infty\right) \text {. }
$$

We conclude by using the left-hand side of (3.24). 
We can now use the fact that $V_{n}^{1}$ and $V_{n}^{2}$ interpret as Fenchel transforms of exponential utility functions, and that the dual probability associated to any exponential utility function is the minimal entropic one $\mathbb{Q}^{e}$, to deduce from (3.23) that the sequence of dual martingale measures $\left(\mathbb{Q}_{n}^{\alpha}\right)_{n \geq 1}$ associated to $\left(U_{n}^{\alpha}\right)_{n \geq 1}$ achieves asymptotically the mininal relative entropy, under Assumption 3.2 and for a suitable choice of the sequence $\left(\alpha_{n}\right)_{n \geq 1}$. We shall see later that this implies convergence to $\mathbb{Q}^{e}$ is the total variation norm.

Lemma 3.2. Let Assumption 3.2 and (3.18) hold. Then, there exists a sequence of positive numbers $\left(\alpha_{n}\right)_{n \geq 1}$ such that

$$
\lim _{n \rightarrow \infty} \mathbb{E}\left[\frac{d \mathbb{Q}_{n}^{\alpha}}{d \mathbb{P}} \log \left(\frac{d \mathbb{Q}_{n}^{\alpha}}{d \mathbb{P}}\right)\right]=\mathbb{E}\left[\frac{d \mathbb{Q}^{e}}{d \mathbb{P}} \log \left(\frac{d \mathbb{Q}^{e}}{d \mathbb{P}}\right)\right] .
$$

Proof. For $i=1,2$, direct computations leads to

$$
\begin{aligned}
\inf _{y>0, \mathbb{Q} \in \mathcal{M}} \mathbb{E}\left[V_{n}^{i}\left(y \frac{d \mathbb{Q}}{d \mathbb{P}}\right)\right] & =\inf _{y>0, \mathbb{Q} \in \mathcal{M}}\left\{\frac{y}{\eta_{n}^{i}} \mathbb{E}\left[\frac{d \mathbb{Q}}{d \mathbb{P}} \log \left(\frac{d \mathbb{Q}}{d \mathbb{P}}\right)\right]+\frac{y}{\eta_{n}^{i}} \log \left(\frac{y}{\alpha_{n}}\right)+\frac{\alpha_{n}-y}{\eta_{n}^{i}}\right\} \\
& =\inf _{y>0}\left\{\frac{y}{\eta_{n}^{i}} \mathbb{E}\left[\frac{d \mathbb{Q}^{e}}{d \mathbb{P}} \log \left(\frac{d \mathbb{Q}^{e}}{d \mathbb{P}}\right)\right]+\frac{y}{\eta_{n}^{i}} \log \left(\frac{y}{\alpha_{n}}\right)+\frac{\alpha_{n}-y}{\eta_{n}^{i}}\right\} \\
& =\mathbb{E}\left[V_{n}^{i}\left(\hat{y}_{n} \frac{d \mathbb{Q}^{e}}{d \mathbb{P}}\right)\right],
\end{aligned}
$$

where the common minimizer $\hat{y}_{n}>0$ is given by

$$
\hat{y}_{n}:=\alpha_{n} e^{-\hat{\rho}} \quad \text { with } \quad \hat{\rho}:=\mathbb{E}\left[\frac{d \mathbb{Q}^{e}}{d \mathbb{P}} \log \left(\frac{d \mathbb{Q}^{e}}{d \mathbb{P}}\right)\right] .
$$

Also note that

$$
\mathbb{E}\left[V_{n}^{2}\left(\hat{y}_{n} \frac{d \mathbb{Q}^{e}}{d \mathbb{P}}\right)-V_{n}^{1}\left(\hat{y}_{n} \frac{d \mathbb{Q}^{e}}{d \mathbb{P}}\right)\right]=\left(1-e^{-\hat{\rho}}\right) \alpha_{n}\left(\frac{1}{\eta_{n}^{2}}-\frac{1}{\eta_{n}^{1}}\right)
$$

so that

$$
\lim _{n \rightarrow \infty} \mathbb{E}\left[V_{n}^{2}\left(\hat{y}_{n} \frac{d \mathbb{Q}^{e}}{d \mathbb{P}}\right)-V_{n}^{1}\left(\hat{y}_{n} \frac{d \mathbb{Q}^{e}}{d \mathbb{P}}\right)\right]=0 \quad \text { whenever } \quad \alpha_{n}\left(\frac{1}{\eta_{n}^{2}}-\frac{1}{\eta_{n}^{1}}\right) \underset{n \rightarrow \infty}{\longrightarrow} 0 .
$$

On the other hand, Lemma 3.1 implies

$$
\mathbb{E}\left[V_{n}^{1}\left(y \frac{d \mathbb{Q}}{d \mathbb{P}}\right)\right] \leq \mathbb{E}\left[V_{n}^{\alpha}\left(y \frac{d \mathbb{Q}}{d \mathbb{P}}\right)\right] \leq \mathbb{E}\left[V_{n}^{2}\left(y \frac{d \mathbb{Q}}{d \mathbb{P}}\right)\right], \quad y>0,
$$

for any $\mathbb{Q} \in \mathcal{M}$. Picking in particular $\mathbb{Q}=\mathbb{Q}_{n}^{\alpha}$, we deduce

$$
\inf _{\mathbb{Q} \in \mathcal{M}} \mathbb{E}\left[V_{n}^{1}\left(y \frac{d \mathbb{Q}}{d \mathbb{P}}\right)\right] \leq \mathbb{E}\left[V_{n}^{1}\left(y \frac{d \mathbb{Q}_{n}^{\alpha}}{d \mathbb{P}}\right)\right] \leq \mathbb{E}\left[V_{n}^{\alpha}\left(y \frac{d \mathbb{Q}_{n}^{\alpha}}{d \mathbb{P}}\right)\right], \quad y>0 .
$$

Taking the infimum over $y>0$ and recalling (3.27), this shows that

$$
\mathbb{E}\left[V_{n}^{1}\left(\hat{y}_{n} \frac{d \mathbb{Q}^{e}}{d \mathbb{P}}\right)\right] \leq \mathbb{E}\left[V_{n}^{1}\left(y_{n} \frac{d \mathbb{Q}_{n}^{\alpha}}{d \mathbb{P}}\right)\right] \leq \inf _{y>0} \mathbb{E}\left[V_{n}^{\alpha}\left(y \frac{d \mathbb{Q}_{n}^{\alpha}}{d \mathbb{P}}\right)\right],
$$

where the minimizer $y_{n}>0$ associated to the middle term is given by

$$
y_{n}:=\alpha_{n} e^{-\rho_{n}} \quad \text { with } \quad \rho_{n}:=\mathbb{E}\left[\frac{d \mathbb{Q}_{n}^{\alpha}}{d \mathbb{P}} \log \left(\frac{d \mathbb{Q}_{n}^{\alpha}}{d \mathbb{P}}\right)\right] .
$$

Similarly, (3.27) and (3.30) imply

$$
\inf _{y>0} \mathbb{E}\left[V_{n}^{\alpha}\left(y \frac{d \mathbb{Q}_{n}^{\alpha}}{d \mathbb{P}}\right)\right]=\inf _{y>0, \mathbb{Q} \in \mathcal{M}} \mathbb{E}\left[V_{n}^{\alpha}\left(y \frac{d \mathbb{Q}}{d \mathbb{P}}\right)\right] \leq \mathbb{E}\left[V_{n}^{2}\left(\hat{y}_{n} \frac{d \mathbb{Q}^{e}}{d \mathbb{P}}\right)\right],
$$


which, combined with (3.31), entails

$$
\mathbb{E}\left[V_{n}^{1}\left(\hat{y}_{n} \frac{d \mathbb{Q}^{e}}{d \mathbb{P}}\right)\right] \leq \mathbb{E}\left[V_{n}^{1}\left(y_{n} \frac{d \mathbb{Q}_{n}^{\alpha}}{d \mathbb{P}}\right)\right] \leq \mathbb{E}\left[V_{n}^{2}\left(\hat{y}_{n} \frac{d \mathbb{Q}^{e}}{d \mathbb{P}}\right)\right] .
$$

If $\alpha_{n}\left(\frac{1}{\eta_{n}^{2}}-\frac{1}{\eta_{n}^{1}}\right) \underset{n \rightarrow \infty}{\longrightarrow} 0,(3.29)$ thus implies that

$$
0 \leq \mathbb{E}\left[V_{n}^{1}\left(y_{n} \frac{d \mathbb{Q}_{n}^{\alpha}}{d \mathbb{P}}\right)-V_{n}^{1}\left(\hat{y}_{n} \frac{d \mathbb{Q}^{e}}{d \mathbb{P}}\right)\right] \underset{n \rightarrow \infty}{\longrightarrow} 0
$$

which, by the definitions in (3.28) and (3.32) of $\hat{y}_{n}$ and $y_{n}$, is equivalent to

$$
\frac{\alpha_{n}}{\eta_{n}^{1}}\left(e^{-\hat{\rho}}-e^{-\rho_{n}}\right) \underset{n \rightarrow \infty}{\longrightarrow} 0 \quad \text { whenever } \alpha_{n}\left(\frac{1}{\eta_{n}^{2}}-\frac{1}{\eta_{n}^{1}}\right) \underset{n \rightarrow \infty}{\longrightarrow} 0 .
$$

We now choose the sequence $\left(\alpha_{n}\right)_{n \geq 1}$ as

$$
\alpha_{n}:=\eta_{n}^{1} \sqrt{\frac{\eta_{n}^{2}}{\eta_{n}^{2}-\eta_{n}^{1}}} \mathbb{1}_{\left\{\eta_{n}^{2} \neq \eta_{n}^{1}\right\}}+\mathbb{1}_{\left\{\eta_{n}^{2}=\eta_{n}^{1}\right\}}, \quad n \geq 1 .
$$

By Assumption 3.2, it satisfies

$\alpha_{n}\left(\frac{1}{\eta_{n}^{2}}-\frac{1}{\eta_{n}^{1}}\right)=\mathbb{1}_{\left\{\eta_{n}^{2} \neq \eta_{n}^{1}\right\}} \sqrt{\frac{\eta_{n}^{1}}{\eta_{n}^{2}}-1} \underset{n \rightarrow \infty}{\longrightarrow} 0$ and $\frac{\alpha_{n}}{\eta_{n}^{1}}=\sqrt{\frac{\eta_{n}^{2}}{\eta_{n}^{2}-\eta_{n}^{1}}} \mathbb{1}_{\left\{\eta_{n}^{2} \neq \eta_{n}^{1}\right\}}+\frac{\mathbb{1}_{\left\{\eta_{n}^{2}=\eta_{n}^{1}\right\}}}{\eta_{n}^{1}} \underset{n \rightarrow \infty}{\longrightarrow} \infty$

Coming back to (3.33), this implies that $\rho_{n} \rightarrow \hat{\rho}$ as $n \rightarrow \infty$, i.e. (3.26) holds.

We are now in position to complete the proof of Theorem 3.2.

Proof of Theorem 3.2. In the following, we let $\left(\alpha_{n}\right)_{n \geq 1}$ be as in Lemma 3.2.

1. We first deduce from Lemma 3.2 that $\left(\mathbb{Q}_{n}^{\alpha}\right)_{n \geq 1}$ converges to $\mathbb{Q}^{e}$ in the norm of total variation. Since $\mathbb{Q}^{e}$ minimizes the entropy with respect to $\mathbb{P}$ over the convex set $\mathcal{M}$, it follows from Theorem 2.2 in [8] that

$$
\mathbb{E}\left[\frac{d \mathbb{Q}}{d \mathbb{P}} \log \left(\frac{d \mathbb{Q}}{d \mathbb{P}}\right)\right] \geq \mathbb{E}\left[\frac{d \mathbb{Q}}{d \mathbb{Q}^{e}} \log \left(\frac{d \mathbb{Q}}{d \mathbb{Q}^{e}}\right)\right]+\mathbb{E}\left[\frac{d \mathbb{Q}^{e}}{d \mathbb{P}} \log \left(\frac{d \mathbb{Q}^{e}}{d \mathbb{P}}\right)\right] \quad \text { for any } \mathbb{Q} \in \mathcal{M}
$$

In particular,

$$
0 \leq \mathbb{E}\left[\frac{d \mathbb{Q}_{n}^{\alpha}}{d \mathbb{Q}^{e}} \log \left(\frac{d \mathbb{Q}_{n}^{\alpha}}{d \mathbb{Q}^{e}}\right)\right] \leq \mathbb{E}\left[\frac{d \mathbb{Q}_{n}^{\alpha}}{d \mathbb{P}} \log \left(\frac{d \mathbb{Q}_{n}^{\alpha}}{d \mathbb{P}}\right)\right]-\mathbb{E}\left[\frac{d \mathbb{Q}^{e}}{d \mathbb{P}} \log \left(\frac{d \mathbb{Q}^{e}}{d \mathbb{P}}\right)\right], \quad n \geq 1
$$

Hence, (3.26) implies that

$$
\mathbb{E}\left[\frac{d \mathbb{Q}_{n}^{\alpha}}{d \mathbb{Q}^{e}} \log \left(\frac{d \mathbb{Q}_{n}^{\alpha}}{d \mathbb{Q}^{e}}\right)\right] \underset{n \rightarrow \infty}{\longrightarrow} 0
$$

The fact that

$$
\mathbb{E}\left[\left|\frac{d \mathbb{Q}_{n}^{\alpha}}{d \mathbb{P}}-\frac{d \mathbb{Q}^{e}}{d \mathbb{P}}\right|\right] \underset{n \rightarrow \infty}{\longrightarrow} 0
$$

then follows from Pinsker's inequality, see e.g. [17].

2. Combining (3.4) and (3.35) implies that

$$
\left|\mathbb{E}^{\mathbb{Q}_{n}^{\alpha}}\left[\frac{G_{n}}{n}\right]-\mathbb{E}^{\mathbb{Q}^{e}}\left[\frac{G_{n}}{n}\right]\right|=\left|\mathbb{E}\left[\frac{G_{n}}{n}\left(\frac{d \mathbb{Q}_{n}^{\alpha}}{d \mathbb{P}}-\frac{d \mathbb{Q}^{e}}{d \mathbb{P}}\right)\right]\right| \leq C \mathbb{E}\left[\left|\frac{d \mathbb{Q}_{n}^{\alpha}}{d \mathbb{P}}-\frac{d \mathbb{Q}^{e}}{d \mathbb{P}}\right|\right] \underset{n \rightarrow \infty}{\longrightarrow} 0,
$$

with $C:=\sup _{n \geq 1}\left\|G_{n} / n\right\|_{L^{\infty}}$. Besides, (2.3) and (3.4) imply that $\mathbb{E}^{\mathbb{Q}^{e}}\left[G_{n} / n\right] \underset{n \rightarrow \infty}{\longrightarrow} \mathbb{E}^{\mathbb{Q}^{e}}[\bar{g}]$. This shows that (3.20) holds. We conclude by using (3.22) and Theorem 3.1. 


\section{References}

[1] D. Bauer, A. Kling, and J. Russ. A universal pricing framework for guaranteed minimum benefits in variable annuities. Astin Bulletin, 38(2):621-651, 2008.

[2] D. Becherer. Rational hedging and valuation of integrated risks under constant absolute risk aversion. Insurance : Mathematics and Economics, 33:1-28, 2003.

[3] B. Bouchard, N. Touzi and A. Zeghal. Dual Formulation of the Utility Maximization Problem : the case of Nonsmooth Utility. Annals of Applied Probability, 14(2):678-717, 2004.

[4] P. Boyle and M. Hardy. Guaranteed annuity options. Astin Bulletin, 33(2):125-152, 2003.

[5] M. J. Brennan and E. S. Schwartz. Alternative investment strategies for the issuers of equity-linked insurance with an asset value guarantee. Journal of Business, 52:63-93, 1979.

[6] M. J. Brennan and E. S. Schwartz. Pricing and investment strategies for guaranteed equitylinked life insurance. Monograph no. 7/The S.S. Huebner Foundation, Wharton School, University of Pennsylvania, 1979.

[7] L. Carassus and M. Rasonyi. Risk-averse asymptotics for reservation price. Annals of Finance, 7(3):375-387, 2011.

[8] I. Csiszár. I-divergence geometry of probability distributions and minimization problems. Ann. Probability, 3:146-158, 1975.

[9] F. Delbaen, P. Grandits, T. Rheinlander, D. Samperi, M. Schweizer, and C. Stricker. Exponential hedging and entropic penalties. Mathematical finance, 12(2):99 - 123, 2002.

[10] F. Delbaen and W. Schachermayer. A general version of the fundamental theorem of asset pricing. Math. Annalen, 300(3):463-520, 1994.

[11] P. Embrechts. Actuarial versus financial pricing of insurance. Journal of Risk Finance, $1(4): 117-146,2000$.

[12] B. K. Goodwin and A. P. Ker. Modeling price and yield risk. A comprehensive assessment of the role of risk in US agriculture, pages 289-323, 2002.

[13] S. D. Hodges and A. Neuberger. Optimal replication of contingent claims under transaction costs. Review of Futures Markets, 8:222 - 239, 1989.

[14] M. A. Milevsky and S. D. Promislow. Mortality derivatives and the option to annuitize. Insurance: Mathematics \& Economics, 29(3):299-318, 2000.

[15] M. A. Milevsky, S. D. Promislow, and V. R. Young. Killing the law of large numbers: Mortality risk premium and the sharpe ratio. The Journal of Risk and Insurance, 73:673686, 2006.

[16] M. Owen. Utility based optimal hedging in incomplete markets. Ann. Appl. Proba., 12:691709, 2002.

[17] F. Piera and P. Parada. On convergence properties of shannon entropy. Problems of Information Transmission, 45:75-94, 2009.

[18] R. Rouge and N. El Karoui. Pricing via utility maximization and entropy. Mathematical Finance, 10(2):259-276, 2000.

[19] W. Schachermayer. Optimal investment when wealth may become negative. Annals of Applied Probability, 11:111 - 177, 1970. 